\title{
Novel geometries and layouts of double-clad fibers for fiber lasers
}

\author{
$\underline{\text { P. Peterka }}^{1,{ }^{*}}$, P. Koška ${ }^{1}$, A. A. Jasim ${ }^{1}$, M. Grábner ${ }^{1}$, J. Aubrecht ${ }^{1}$, M. Kamrádek ${ }^{1,2}$, \\ O. Podrazký ${ }^{1}$, I. Bartoń ${ }^{1}$, F. Todorov ${ }^{1}$, Nithyanandan Kanagaraj ${ }^{1,3}$, \\ I. Kašík ${ }^{1}$ and P. Honzátko ${ }^{1}$ \\ ${ }^{I}$ Institute of Photonics and Electronics of the Czech Academy of Sciences \\ ${ }^{2}$ Faculty of Nuclear Sciences and Physical Engineering, Czech Technical University in Prague \\ ${ }^{3}$ now in Optoelectronic Research Centre, University of Southampton, United Kingdom \\ "E-mail: peterka@ufe.cz
}

DOI: 10.31868/RFL2020.45-46

High-power operation of fiber lasers was mainly enabled by the invention of cladding-pumping in a double-clad fiber structure. Pump absorption is enhanced by broken circular symmetry of inner cladding cross-sections and by mode-scrambling of the pump modes resulting from unconventional fiber coiling, e. g., twisted fiber on standard spool [1, 2], spiral shape coil [3] or kidney shape coil with concave and convex sections of the coil shape [4], see Fig. 1 and 2. However, theoretical studies were mostly limited to the assumption of a straight fiber until recently, when the rigorous model accounting for double-clad fiber bending and twisting was described $[1,2,5]$. Here we review the results of numerical modeling of pump absorption in various types of double-clad fibers, e. g., with cross-section shape of hexagon, stadium, and circle; two-fiber bundle (so-called GT Wave fiber structure, see Fig. 3) as well as panda fibers. We analyzed both the cases: of diode pumping where the ratio of core area to inner cladding area is usually small, and the case of tandem pumping by high-brightness fiber laser sources, where the respective ratio is usually larger. We show also our results in processing of fiber preforms by $\mathrm{CO}_{2}$ laser in order to get almost arbitrary cross sectional shape of the inner cladding [6]. Examples of such fiber cross sections are shown in Fig. 1. Unlike the common grinding method, $\mathrm{CO}_{2}$ laser shaping allows to achieve almost any cross section shape, namely it allows combination of concave and convex sections.

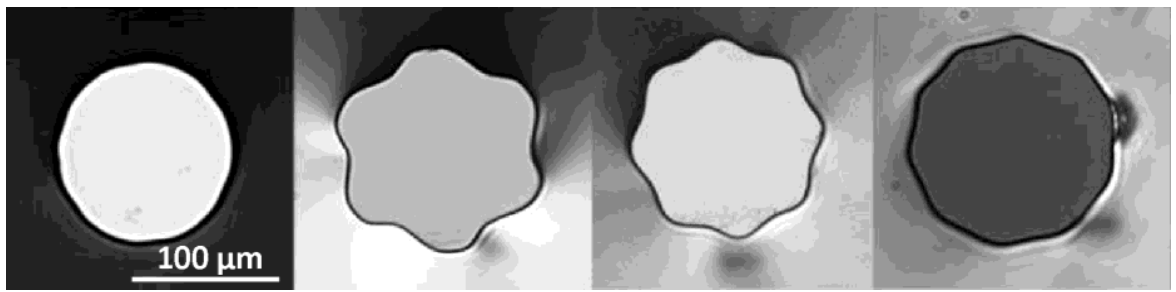

Fig. 1. Examples of noncircular fiber cross sections whose silica preforms were shaped by $\mathrm{CO}_{2}$ laser. From left: hexagonal shapes with shallow and deeper groves, octagonal and decagonal cross sections.

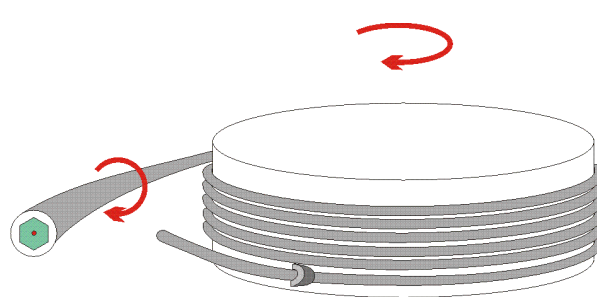

(a)

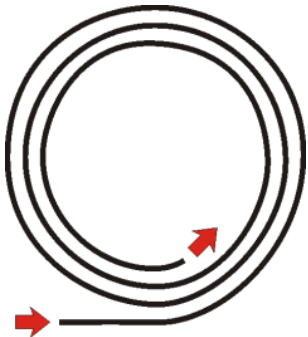

(b)

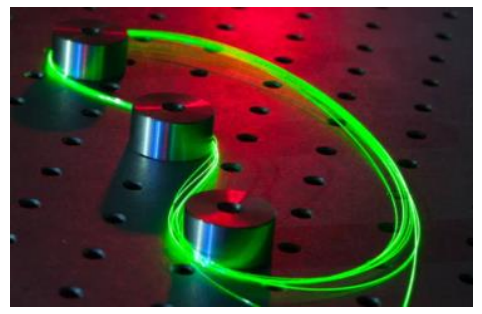

(c)

Fig. 2. Examples of fiber layout for improvement of pump absorption efficiency: (a) twisted fiber on a standard circular spool, (b) spiral spool and (c) kidney-shaped spool. The spiral spool offers slowly vary- 
ing effective absorption cross section through change of the coiling diameter. Used with permission from ref. [7] (C[2019] IEEE)

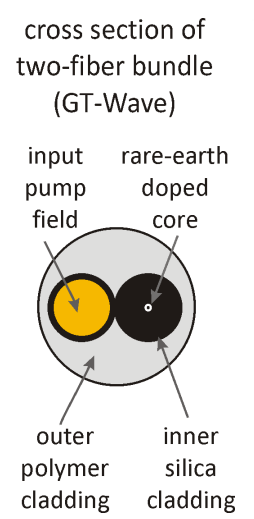

(a)

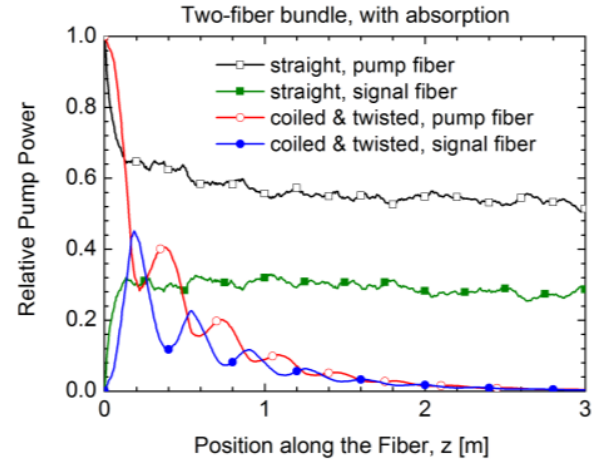

(b)

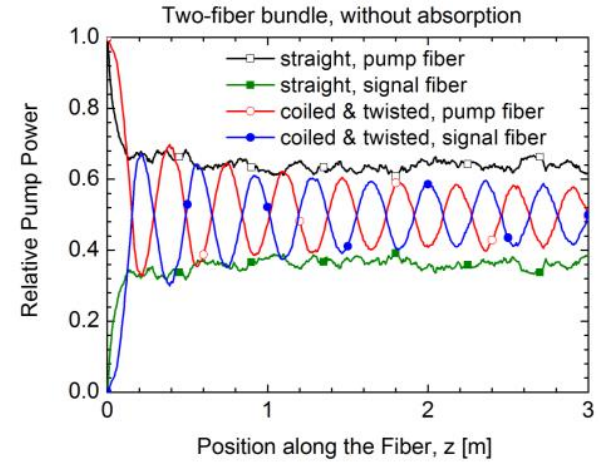

(c)

Fig. 3. (a) Cross section of the two-fiber bundle double-clad waveguide structure; the yellow part schematically represents the input pump distribution. The pump power distribution between both fibers in GT-Wave two-fiber bundle was numerically calculated either for the case of straight arrangement orfor coiled and twisted fiber bundle. Two cases of signal fiber are considered, (a) without rare-earth dopants and (b) with Yb-doped core. Used with permission from ref. [1] (C[2016] IEEE)

Our model used for pump absorption efficiency optimization (against other published models which do not take into account fiber twist) has a significant application potential in the design of fiber lasers and amplifiers because double-clad fibers of shorter lengths can be used $[1,2]$. This minimizes the deleterious effect of background losses and nonlinear effects. In addition, our model finally explained highly efficient pump coupling in GT-Wave fiber structure where the pump fiber is only touching the fiber with the active core, see Fig. 3(a). While only negligible absorption occurs in straight GT-Wave structure, see green line in Fig. 3(b), it is the twist of the fiber bundle that enables efficient pump absorption, see the blue line in Fig. 3(b) [1].

The new geometries and layouts shall finally result in a highly efficient laser of small footprint without the need of water cooling and holds great potential for applications with low power consumption, tightly limited space and weight requirements. The proposed design will also minimize the risk of damage of the fiber during laser operation, which is a key point in fiber based laser architecture. Portions of the work have already been presented in papers $[1,2,7]$.

This work was financially supported by the Czech Science Foundation under project No. 19-03141S.

\section{References}

[1] P. Koska, P. Peterka, and V. Doya, IEEE J. Sel. Top. Quantum Electron. 22, 4401508 (2016).

[2] P. Koska, et al., Opt. Express 24, 102-107 (2016).

[3] C. A. Codemard, A. Malinowski, and M. N. Zervas, in Proc. SPIE Photonics West, Fiber Lasers XIV: Technology and Systems, San Francisco, USA, 2017, p. 1008315.

[4] H. Zellmer, et al., in Proc. Opt. Amplifiers and Their Appl., Victoria, Canada, 1997, p. FAW18.

[5] P. Koska and P. Peterka, Opt. Quantum Electron. 47, 3181-3191 (2015).

[6] A. A. Jasim, et al., Opt. Express 28, 13601-13615 (2020).

[7] P. Peterka, et al., in IEEE Proc. $21^{\text {st }}$ International Conference on Transparent Optical Networks (ICTON), Angers, France, 2019, p. Th.B6.1. 